\title{
Perceived Discrimination, Depressive Symptoms, and Substance Use in Young Adulthood
}

\author{
Trenette T. Clark, PhD, LCSW \\ University of North Carolina at Chapel Hill 325 Pittsboro St., CB\# 3550 Chapel Hill, NC USA \\ 27599 ttclark@email.unc.edu (919) 843-8020
}

\section{Abstract}

Perceived discrimination is an important health-related stressor. As suggested by the stress-coping model, substance use often serves as a means to reduce the negative effects of perceived discrimination. This study uses data from the National Survey of American Life-Adults to examine the structural relationship of perceived discrimination and depressive symptoms with lifetime and recent substance use among African American and African Caribbean young adults. Respondents $(N=1,910)$ were 18-35 years old. Compared with African Caribbeans, African Americans report significantly higher levels of depressive symptoms and both lifetime and recent substance use. Multiple-group structural equation modeling is used to evaluate model fit and test hypothesized models. Results show good fit of the hypothesized models in both African Americans and African Caribbeans. Full measurement and structural invariance is found across ethnicity. Mediation models explain $18.5 \%$ and $47.4 \%$ of the variance in lifetime substance use for African Americans and African Caribbeans, respectively, and $23.5 \%$ and $35.0 \%$ of the variance in recent substance use for African Americans and African Caribbeans, respectively. Mediation tests indicate depressive symptoms partially mediate the relationship between perceived discrimination and lifetime substance use and fully mediated this relationship for recent substance use. This study is the first to demonstrate a positive association between perceived racial discrimination and substance use among African Caribbean young adults. Study findings illuminate the influence of perceived discrimination on substance use and the mechanisms of this relationship among African American and African Caribbean young adults.

\section{Keywords}

Black; drug; cigarette; alcohol; marijuana; depression

\footnotetext{
(C) 2014 Elsevier Ltd. All rights reserved.

Publisher's Disclaimer: This is a PDF file of an unedited manuscript that has been accepted for publication. As a service to our customers we are providing this early version of the manuscript. The manuscript will undergo copyediting, typesetting, and review of the resulting proof before it is published in its final citable form. Please note that during the production process errors may be discovered which could affect the content, and all legal disclaimers that apply to the journal pertain.

Author Disclosures

This manuscript is original research (i.e., does not contain data that is currently submitted or published), has been submitted solely to Addictive Behaviors, has not been previously published, nor has its findings been posted online. If accepted for publication, it will not be published elsewhere. The work in its entirety is in compliance with ethical standards governing the treatment of human subjects. I declare no conflict of interest.
} 


\section{Introduction}

Despite ongoing prevention efforts, substance use continues to be a prevalent concern with persistent social and health consequences, especially the racial/ethnic disparities in substance use health outcomes. Research has consistently shown that as compared with White and Hispanics counterparts, Black adolescents and young adults generally report a greater number of negative substance-use related consequences (Gil, Wagner, \& Tubman, 2004; Mulia, Ye, Greenfield, \& Zemore, 2009). For example, a longitudinal study with a representative sample of the U.S. population of adults who transitioned from abstainers during early adolescence to regular use of substances in young adulthood found that African Americans were 6.6 times more likely to experience a substance-use disorder compared to Whites (4.3) and Hispanics (2.8) (Gil et al., 2004).

During young adulthood, the advantageous lower rates of substance use found in Black adolescents are lost and their rates of substance-use become comparable to their White and Hispanic counterparts (Clark, Corneille, \& Coman, 2013; Geronimus, Neidert, \& Bound, 1993). Although the causes of this phenomenon, referred to as the "catch-up effect," are unknown, perceived discrimination may be an important predictor of the catch-up effect. By adulthood and as compared to Asian Americans, Puerto Ricans, and Latinos, Black adolescents tend to report steeper increases of perceived racial discrimination (Greene, Way, $\&$ Pahl, 2006). Thus, for some Blacks the cumulative effects of discrimination can lead to new substance use in young adulthood, perhaps helping to explain the catch-up effect.

Indeed, discrimination is a powerful source of health-related stress for young adults (Whitbeck, Hoyt, McMorris, Chen \& Stubben, 2001), with its magnitude ranking alongside major negative events such as death of a loved one, divorce, and job loss (Kessler, Mickelson, \& Williams, 1999). One pathway to poor physical health is through discrimination's effect on substance use. Research supports an association between perceived discrimination and substance use (Brody, Kogan, \& Chen, 2012), including tobacco (Bennett, Wolin, Robinson, Fowler, \& Edwards, 2005), alcohol (Terrell, Miller, Foster, \& Watkins, 2006), and marijuana (Choi, Harachi, Gillmore, \& Catalano, 2006). Several longitudinal studies have examined the direction of the relationship between discrimination and substance use, concluding discrimination causes substance use and not the reverse (Brody et al., 2012; Gibbons et al., 2010; Gibbons, Gerrard, Cleveland, Willis, \& Brody, 2004).

The stress-coping model, which has framed much of the research on discrimination, posits that perceived discrimination depletes coping resources and encourages avoidant coping strategies such as anger, frustration, sadness, and depression (Clark, Anderson, Clark, \& Williams, 1999). Empirical studies support the stress-coping model by suggesting discrimination leads to avoidance and internalizing symptoms such as depressive symptoms (Kessler et al., 1999). These findings are consistent with others who found discrimination was indirectly related to substance use among African American adolescents and young adults and mediated by depressive symptoms (e.g., Brody et al., 2012). 
Guided by the stress-coping model, this study proposed that African American and African Caribbean young adults turn to substance use as a means to reduce depressive symptoms stemming from perceived discrimination. Because country and culture-of-origin can contribute to variations in manifestations of discrimination, stress, and coping (Georgiades, Boyle, \& Duku, 2007; Rong \& Brown, 2002), such differences are expected between African Americans and African Caribbeans. The current study used data from the National Survey of American Life-Adult (NSAL) database and multiple group structural equation modeling (1) to investigate the process through which perceived everyday discrimination affects lifetime and recent substance use, and (2) to assess whether the mediated model is moderated by ethnicity.

\section{Method}

\subsection{Data Source and Study Sample}

The sample was drawn from 6,082 adults in the NSAL. NSAL includes a large, nationally representative sample of 3,570 African Americans, 1,621 African Caribbeans, and 891 nonHispanic Whites. Most surveys were administered in face-to-face interviews in respondents' homes using a computer-assisted instrument. Respondents' age range was 18-94 years ( $M=$ 43.22 years; $S D=.21$ ). Details of the NSAL sample and data collection are available elsewhere (Jackson et al., 2004). Inclusion criteria limited the study sample to adults 18-35 years who self-identified as African American or African Caribbean, yielding an analytic sample of 1,910 young adults.

\subsection{Measurement of Independent Variables}

2.2.1 Ethnicity—Respondents self-identified as African American or African Caribbean.

2.2.2 Perceived everyday discrimination-Perceptions of discrimination were measured using the 10-item Everyday Discrimination scale that assesses routine experiences of unfair treatment (Williams, Yu, Jackson, \& Anderson, 1997). The 10 items asked respondents how often they perceived experiencing discrimination in the following routine scenarios: treated with less courtesy than others (PD1); treated with less respect (PD2); receiving poorer service at restaurants/stores (PD3); treated as less smart (PD4); people acting afraid of them (PD5); treated as dishonest (PD6); people acting as if they are better than them (PD7); called names or insulted (PD8); threatened or harassed (PD9); and followed in stores $(\mathrm{PD} 10)$. The scale had a high internal reliability (Cronbach alpha $=.89$ ).

2.2.3 Discrimination attribution-One item assessed the primary reason for discrimination (i.e., race, ethnicity, shade of skin color, gender, age, height and weight, other).

2.2.4 Depressive symptoms-Depressive symptoms were assessed using a12-item version of the Center for Epidemiological Studies-Depression (CES-D) scale (Roberts \& Sobhan, 1992). The CES-D measures the extent during the past week that respondents experienced the following feelings or moods: as good as other people (DS1); trouble keeping mind on tasks (DS2); depressed (DS3); everything an effort (DS4); hopeful (DS5); 
restless sleep (DS6); happy (DS7): people were unfriendly (DS8); enjoyed life (DS9); crying spells (DS10); others disliked them (DS11); and could not get going (DS12). A reliability analysis yielded a Cronbach's alpha of .77.

\subsection{Measurement of Dependent Variables}

2.3.1 Lifetime Substance Use-Two dichotomous items (yes $=1 ; n o=0$ ) asked respondents if they had ever smoked marijuana or smoked more than 100 cigarettes in their lifetime. One item asked the age at which respondents first drank alcohol; responses were collapsed to yes $(=1)$ for those who reported an age, and no $(=0)$ for those who reported never drinking alcohol.

2.3.2 Recent substance use-Respondents reported the frequency during the past 12 months with which they smoked marijuana or drank alcohol.

\subsection{Measurement of Control variables}

The models incorporated covariates shown in other research to be associated with substance use, including age, gender, income, education, employment status, marital status, and nativity.

\subsection{Analytic Strategy}

Structural equation model (SEM) analyses were conducted in Mplus 7.11 (Muthén \& Muthén, 1998-2012) using the mean and variance-adjusted weighted least squares (WLSMV) estimator, a recommended robust WLS estimator (Muthén, du Toit, \& Spisic, 1997) for nonnormal and categorical data. Variables for substance-use outcomes were specified as categorical variables. The analyses controlled for the complex sampling design by using the complex sampling procedure in Mplus and by including cluster, stratum, and weighting variables in all analyses. The small percentage of missing data was minimized by using pairwise calculation of the covariances. Goodness of model fit was evaluated using chi-square and its $p$-value, the comparative fit index (CFI), the Tucker-Lewis index (TLI), and the root mean square error of approximation (RMSEA). To assess measurement and structural invariance, the DIFFTEST command in Mplus was used to obtain correct chisquare difference tests between baseline and nested models (Muthén \& Muthén, 1998-2012).

\section{Results}

\subsection{Descriptive Statistics}

The analytic sample consisted of 1,910 African American (69.1\%) and African Caribbean (30.9\%) young adults $(64.7 \%)$. The mean age was $26.9(S D=.12)$ years. $T$ tests revealed that African American young adults reported significantly higher levels of both lifetime and recent substance use, and depressive symptoms compared with African Caribbean young adults $(p<0.01)$. No significant differences were found in discrimination experiences.

\subsection{Multiple-group SEM}

3.2.1 Measurement and Structural Invariance-Measurement invariance tests indicated factor loadings and indicators were invariant across groups for both lifetime and 
recent substance use ( $p>.05)$, suggesting the factor loadings are the same for African American and African Caribbean young adults. These findings established full measurement invariance. Thus, the next step was to test structural invariance. For both the lifetime and the recent substance-use models, the DIFFTEST's $\chi^{2}$ for the nested models with structural path constraints was not significant $(p>.05)$ suggesting the structural models are invariant. Therefore, the final models with measurement and structural constraints were retained and reported as the final SEM models.

\subsection{Final SEM Models}

3.3.1 Lifetime substance use-The proposed model had a very good fit to the data, $\chi^{2} / d f(1381.29 / 1097), p=.000 ;$ CFI=.956; TFI=.956; RMSEA=.017 (.014, .019). The chisquare test suggested the model fit the data slightly better for African Caribbeans $\left(\chi^{2}=650.10\right)$ than African Americans $\left(\chi^{2}=731.19\right)$. Figure 1 presents a diagram of the lifetime substance-use model. Controlling for the covariates, discrimination was positively associated with depressive symptoms for African Americans and African Caribbeans $(p<$. $01)$. The standardized coefficients indicated a slightly stronger relationship among African Americans than African Caribbeans (.413, .388, respectively). Mediation tests indicated that depressive symptoms partially mediated the relationship between perceived discrimination and lifetime substance use for African American and African Caribbean young adults ( $p<$. 01 ). The standardized coefficients indicated this mediated relationship was slightly stronger for African Americans than African Caribbeans (.131, .105, respectively). The model (perceived discrimination and depressive symptoms) explained 18.5\% of the variance of lifetime substance use for African American and $47.4 \%$ of the variance for African Caribbean young adults. Perceived discrimination explained $28.5 \%$ of the variance of depressive symptoms for African Americans and 36.8\% of the variance for African Caribbean young adults.

3.3.2 Recent Substance Use-The proposed model provided a very good fit to the data, $\chi^{2} / d f(1310.661 / 1053), p=.000$; CFI=.96; TFI=.96; RMSEA=.016 $(.013, .019)$. The chisquare test suggested the model fit the data slightly better for African Caribbean $\left(\chi^{2}=635.51\right)$ than African American young adults $\left(\chi^{2}=675.15\right)$. Figure 2 illustrates the recent substance-use model. Discrimination was positively associated with depressive symptoms for African Americans and African Caribbeans $(p<.01)$. The standardized coefficients indicated this relationship was slightly stronger for African Americans than African Caribbeans (.411, .382, respectively). Mediation tests indicated depressive symptoms fully mediated the relationship between perceived discrimination and recent substance use among African American and African Caribbean young adults $(p=.06)$. The standardized coefficients indicated this mediated relationship was slightly stronger for African Americans than African Caribbeans (.085, .079, respectively). The model explained 23.5\% of the variance of recent substance use for African American young adults and 35.0\% for African Caribbean young adults. Perceived discrimination explained 27.7\% of the variance of depressive symptoms for African Americans and 37.5\% of the variance for African Caribbean young adults. 


\section{Discussion}

Grounded in stress-coping theory, this study is the first to demonstrate that perceived racial discrimination is positively associated with lifetime and recent substance use among African Caribbean young adults. Similarly, the findings supported prior research showing an association of perceived discrimination with substance use among African American young adults (Bennett et al., 2005; Choi et al., 2006; Terrell et al., 2006). Mediation models explained nearly half (47.4\%) of the variance in lifetime substance use for African Caribbeans and nearly one-fifth (18.5\%) of the variance for African Americans. Mediation models also explained over one-third (35\%) and nearly one-fourth $(23.5 \%)$ of the variance in recent substance use for African Caribbean and African American young adults, respectively. These moderate-to-large effect sizes (Cohen, 1992) suggest the models are promising for understanding substance use within the Black population subgroups, especially among African Caribbeans.

This study presents two key findings: 1) the measurement and structural relationships between discrimination, depressive symptoms, and substance use are invariant across ethnicity; and 2) depressive symptoms partially mediated the relationship between perceived discrimination and lifetime substance use, while fully mediating the relationship between perceived discrimination and recent substance use. This study provides compelling preliminary evidence suggesting African American and African Caribbean young adults cope with discrimination by exhibiting depressive symptoms and engaging in substance use. An important direction for future research is to examine these relationships using repeated measures, harder drugs, and assessing externalizing reactions (e.g., anger, hostility) as potential mediators.

\section{Acknowledgments}

This work was supported by funds from an AAUW American Fellowship Research Publication Grant and the National Institutes of Health/National Institute on Drug Abuse (1K01DA035895-01) awarded to the author.

\section{References}

Bennett GG, Wolin KY, Robinson EL, Fowler S, Edwards CL. Perceived racial/ethnic harassment and tobacco use among African American young adults. American Journal of Public Health. 2005; 95(2):238-240. http://dx.doi.org/10.2105/AJPH.2004.037812. [PubMed: 15671457]

Brody GH, Kogan SM, Chen Y. Perceived discrimination and longitudinal increases in adolescent substance use: Gender differences and mediational pathways. American Journal of Public Health. 2012; 102(5):1006-1011. http://dx.doi.org/10.2105/AJPH.2011.300588. [PubMed: 22420807]

Choi Y, Harachi TW, Gillmore MR, Catalano RF. Are multiracial adolescents at greater risk? Comparisons of rates, patterns, and correlates of substance use and violence between monoracial and multiracial adolescents. American Journal of Orthopsychiatry. 2006; 76:86-97. http:// dx.doi.org/10.1037/0002-9432.76.1.86. [PubMed: 16569131]

Clark R, Anderson NB, Clark VR, Williams DR. Racism as a stressor for African Americans: A biopsychosocial model. American Psychologist. 1999; 54(10):805-816. http://dx.doi.org/ 10.1037/0003-066X.54.10.805. [PubMed: 10540593]

Clark TT, Corneille M, Coman E. Developmental trajectories of alcohol use among monoracial and biracial Black adolescents and adults. Journal of Psychoactive Drugs. 2013; 45(3):249-257. http:// dx.doi.org/10.1080/02791072.2013.805980. [PubMed: 24175490] 
Cohen J. A power primer. Psychological Bulletin. 1992; 112(1):155-159. http://dx.doi.org/ 10.1037/0033-2909.112.1.155. [PubMed: 19565683]

Georgiades K, Boyle MH, Duku E. Contextual influences on children's mental health and school performance: The moderating effects of family immigrant status. Child Development. 2007; 78(5): 1572-1591. http://dx.doi.org/10.1111/j.1467-8624.2007.01084.x. [PubMed: 17883449]

Geronimus AT, Neidert LJ, Bound J. Age patterns of smoking in U.S. Black and White women of childbearing age. American Journal of Public Health. 1993; 83(9):1258-1264. http://dx.doi.org/ 10.2105/AJPH.83.9.1258. [PubMed: 8363001]

Gibbons FX, Etcheverry PE, Stock ML, Gerrard M, Weng CY, Kiviniemi M, O'Hara RE. Exploring the link between racial discrimination and substance use: What mediates? What buffers? Journal of Personality and Social Psychology. 2010; 99(5):785-801. http://dx.doi.org/10.1037/a0019880. [PubMed: 20677890]

Gibbons FX, Gerrard M, Cleveland MJ, Wills TA, Brody G. Perceived discrimination and substance use in African American parents and their children: A panel study. Journal of Personality and Social Psychology. 2004; 86(4):517-529. http://dx.doi.org/10.1037/0022-3514.86.4.517. [PubMed: 15053703]

Gil AG, Wagner EF, Tubman JG. Associations between early-adolescent substance use and subsequent young-adult substance use disorders and psychiatric disorders among a multiethnic male sample in South Florida. American Journal of Public Health. 2004; 94(9):1603-1609. http:// dx.doi.org/10.2105/AJPH.94.9.1603. [PubMed: 15333322]

Greene ML, Way N, Pahl K. Trajectories of perceived adult and peer discrimination among Black, Latino, and Asian American adolescents: Patterns and psychological correlates. Developmental Psychology. 2006; 42(2):218-236. http://dx.doi.org/10.1037/0012-1649.42.2.218. [PubMed: 16569162]

Jackson JS, Torres M, Caldwell CH, Neighbors HW, Nesse RM, Taylor RJ, Williams DR. The National Survey of American Life: A study of racial, ethnic and cultural influences on mental disorders and mental health. International Journal of Methods in Psychiatric Research. 2004; 13(4):196-207. http://dx.doi.org/10.1002/mpr.177. [PubMed: 15719528]

Kessler, RC.; Mickelson, KD.; Williams, DR. The prevalence, distribution, and mental health correlates of perceived discrimination in the United States.; Journal of Health and Social Behavior. 1999. p. 208-230.http://dx.doi.org/10.2307/2676349

Mulia N, Ye Y, Greenfield TK, Zemore SE. Disparities in alcohol-related problems among White, Black, and Hispanic Americans. Alcoholism: Clinical and Experimental Research. 2009; 33(4): 654-662. http://dx.doi.org/10.1111/j.1530-0277.2008.00880.x.

Muthén, LK.; Muthén, BO. Mplus user's guide. 7th ed.. Muthén \& Muthén; Los Angeles, CA: 1998-2012.

Muthén, B.; du Toit, SH.; Spisic, D. Robust inference using weighted least squares and quadratic estimating equations in latent variable modeling with categorical and continuous outcomes [Technical report]. 1997. Retrieved from http://pages.gseis.ucla.edu/faculty/muthen/articles/ Article_075.pdf

Roberts RE, Sobhan M. Symptoms of depression in adolescence: A comparison of Anglo, Africans, and Hispanic Americans. Journal of Youth and Adolescence. 1992; 21:639-651. http://dx.doi.org/ 10.1007/BF01538736. [PubMed: 24264167]

Rong XL, Brown F. Socialization, culture, and identities of Black immigrant children: What educators need to know and do. Education and Urban Society. 2002; 34(2):247-273. http://dx.doi.org/ 10.1177/0013124502342008.

Terrell F, Miller AR, Foster K, Watkins CE Jr. Racial discrimination-induced anger and alcohol use among Black adolescents. Adolescence. 2006; 41:485-492. [PubMed: 17225663]

Whitbeck, LB.; Hoyt, DR.; McMorris, BJ.; Chen, X.; Stubben, JD. Perceived discrimination and early substance abuse among American Indian children.; Journal of Health and Social Behavior. 2001. p. 405-424.http://dx.doi.org/10.2307/3090187

Williams DR, Yu Y, Jackson JS, Anderson NB. Racial differences in physical and mental health: Socioeconomic status, stress, and discrimination. Journal of Health Psychology. 1997; 2(3):335351. http://dx.doi.org/10.1177/135910539700200305. [PubMed: 22013026] 


\section{Highlights}

- African Americans reported higher levels of substance use than African Caribbeans

- African Americans reported higher levels of depressive symptoms than African Caribbeans

- Full measurement and structural invariance was found across ethnicity

- Depressive symptoms partially mediated discrimination and lifetime substance use

- Depressive symptoms fully mediated discrimination and recent substance use 


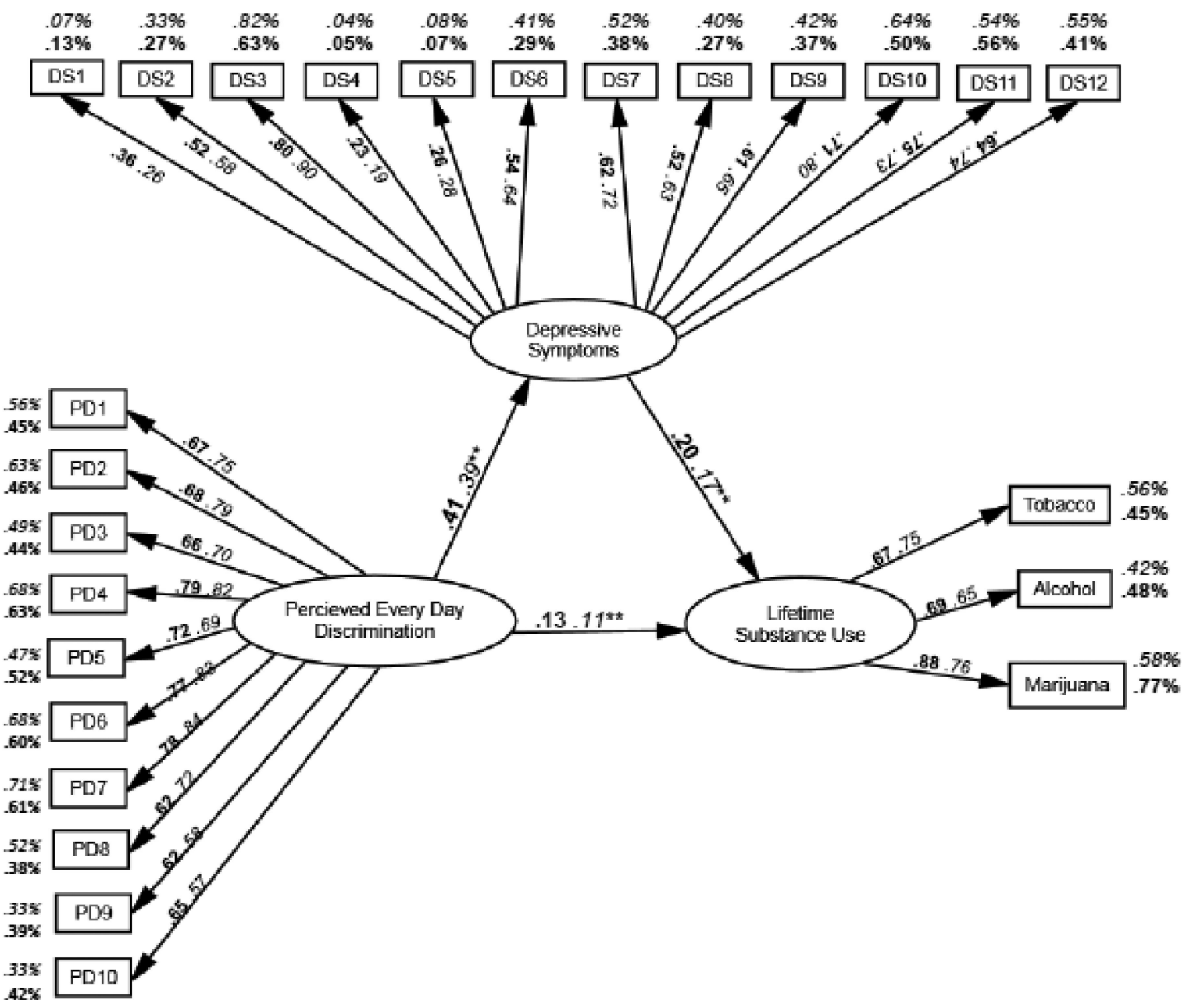

Figure 1.

Measurement and Structural Model of Perceived Discrimination's Indirect Effect on Lifetime Substance Use among African American and Afro-Caribbean Young Adults. 


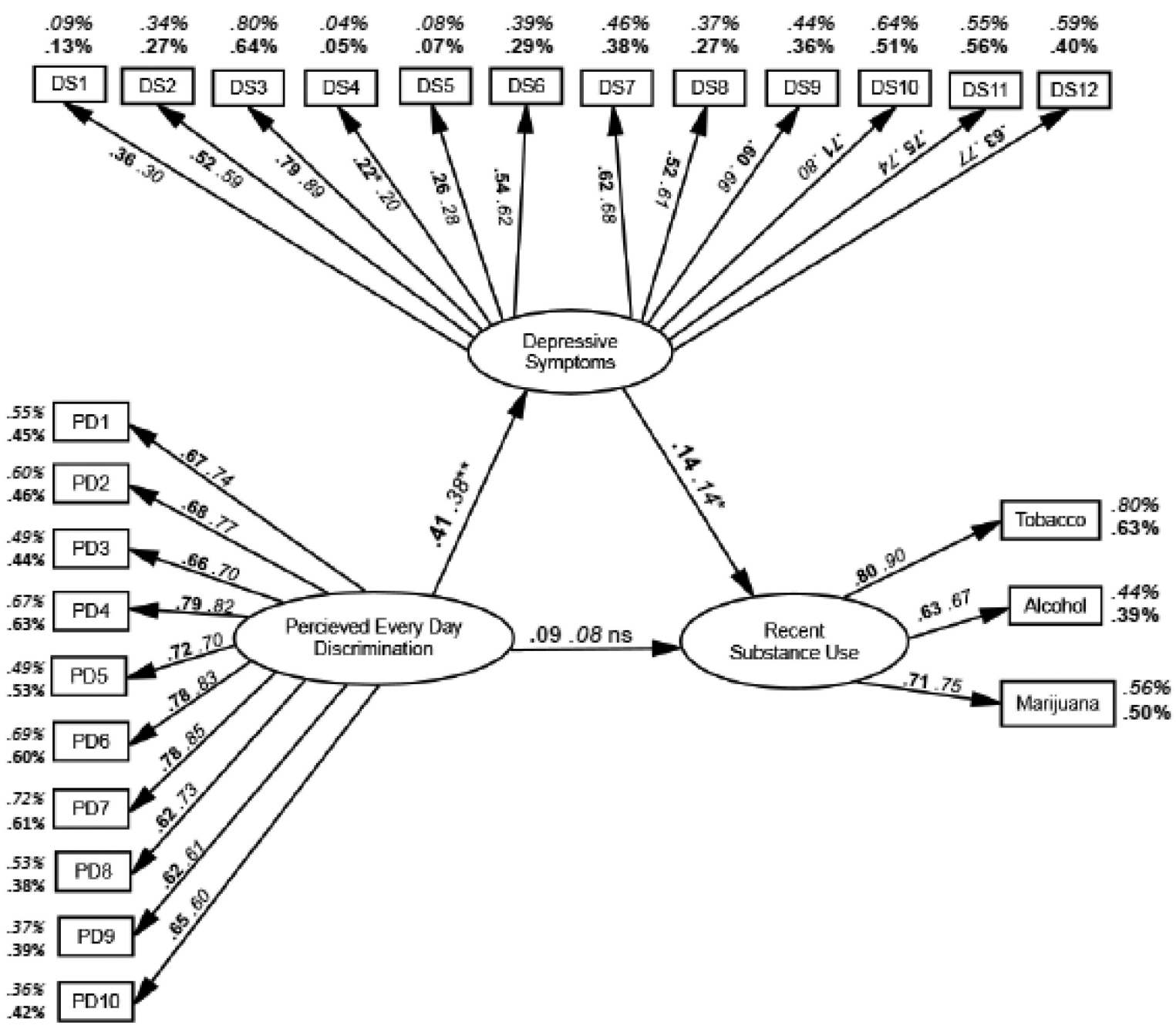

Figure 2.

Measurement and Structural Models for Reecent Substance Use among African American and Afro-Caribbean Young Adults. 